\title{
GRUPO DE APOIO PARA FAMILIARES DE PESSOAS COM AUTISMO: RELATO DE EXPERIÊNCIA
}

\author{
SUPPORT GROUP FOR FAMILY PEOPLE WITH AUTISM: \\ EXPERIENCE REPORT
}

\author{
Josilene do Nascimento Rodrigues ${ }^{1}$
} Débora Priscila Ferreira da Silva Gomes ${ }^{2}$

\begin{abstract}
RESUMO: O diagnóstico de autismo para uma família constitui grande impacto, modificando o contexto de vida dos sujeitos que convivem com a pessoa com autismo, uma vez que ocasiona mudanças na rotina diária, na dinâmica familiar, no meio social e financeiro, tornando-se importante envolver a família no processo de cuidado e intervenção. Este estudo descritivo do tipo relato de experiência tem como objetivo descrever a experiência apreendida, enquanto psicóloga residente, em um grupo de apoio familiar. Utilizou como instrumentos para coleta a observação e o diário de campo, sendo analisada a partir da análise de conteúdo de Bardin. Constatou-se que o grupo promove o acolhimento, o suporte nas dificuldades, o compartilhamento de sentimentos e a interação entre profissional-familiar. Portanto, o grupo tem potencial terapêutico, pois incita o cuidado.
\end{abstract}

Palavras chave: Autismo; família; cuidado.

ABSTRACT: The diagnosis of autism for a family constitutes a major impact, changing the context of life of the subjects who live with the person with autism, since it causes changes in the daily routine, in the family dynamics, in the social and financial environment, making it important to involve the family in the care and intervention process. This descriptive study of the experience report type aims to describe the experience learned, as a resident psychologist, in a family support group. It used as instruments for collection the observation and the field diary, being analyzed from the content analysis of Bardin. It was found that the group promotes

\footnotetext{
1 Graduada em Psicologia pela Universidade Federal da Campina Grande, Especialista na modalidade de Residência Multiprofissional em Saúde da Família e Comunidade da Faculdade de Ciências Médicas da Paraíba, Mestranda em Psicologia da Saúde pela Universidade Estadual da Paraíba. Endereço eletrônico: lene-nr@hotmail.com.

${ }^{2}$ Graduada em Psicologia, Mestranda em Psicologia da Saúde pela Universidade Estadual da Paraíba, Especialista em Saúde Coletiva (UNIFIP), Especialista em Educação em Direitos Humanos (UFPB). Endereço eletrônico: debora_priscila@hotmail.com.
} 
welcoming, support in difficulties, sharing feelings and interaction between professional and family. Therefore, the group has therapeutic potential, as it encourages care.

Keywords: Autism; family; caution. 


\section{INTRODUÇÃO}

O Autismo também chamado de Transtorno do Espectro Autista (TEA) apresenta-se como uma síndrome do comportamento que afeta o desenvolvimento psicomotor e neurológico, comprometendo significativamente a cognição, a comunicação e a interação social. As manifestações clínicas do TEA aparecem antes dos 36 meses de idade e tornam-se mais evidentes, especialmente, quando a criança é inserida nos espaços sociais (PINTO, et al., 2016; MELLO, 2007).

No Manual Diagnóstico e Estatístico de Transtornos mental - DSM-V (2014), o TEA é descrito como um transtorno do Neurodesenvolvimento. De acordo com Mello (2007) as causas ainda são desconhecidas, mas, hodiernamente é considerada como uma síndrome de origem multicausal, a qual pode envolver fatores variados como o genético, neurológico, psicossocial e o ambiental.

Estudo divulgado pelo Center for Disease Control, revela que uma a cada 68 crianças nascem com TEA, ou seja, pode atingir $1,47 \%$ dos indivíduos da população mundial. No Brasil, ainda não existem estudos conclusivos que apresentam a prevalência do TEA, mas segundo a Federação Nacional das Apaes, estima-se que exista cerca de dois milhões de autistas, e que os mais afetados são crianças do sexo masculino (CENTERS FOR DISEASE CONTROL AND PREVENTION, 2010; APAE, 2017; MELLO, et al., 2013).

Existem poucos estudos epidemiológicos sobre o autismo, mas observam-se avanços nas últimas décadas em relação ao diagnóstico precoce, devido o maior conhecimento das manifestações clínicas e ao surgimento de serviços especialidades em TEA (PINTO, et al., 2016). Entretanto, um dos principais problemas é definir um diagnóstico de autismo, visto que não tem um marcador biológico que pode ser identificado por exames laboratoriais.

Apesar de o autismo ser de difícil diagnóstico, existem alguns sinais que podem indicar a presença de traços autistas e que podem ser percebidos no contexto familiar, escolar e social. Estes sinais podem ser: a presença de 
comportamentos, interesses e atividades estereotipadas, repetitivas e restritas, a resistência para mudança de rotina, as crises de agressividade, a não demonstração de envolvimento afetivo e de contato visual, dificuldade na comunicação, na interação social, assim como, padrão de inteligência variável e de temperamento lábil (FEDERAÇÃO NACIONAL DAS APAES, 2017; DSM-5, 2014; ABRA, 2013).

Outras condições do neurodesenvolvimento ou comportamental devem ser observadas, tais como a hiperatividade, o déficit de atenção, os comportamentos autoagressivos e autodestrutivos, distúrbios de humor e de afeto (ABRA, 2013; DSM-5, 2014).

É necessário realizar uma avaliação complexa, que deve ser feita por uma equipe de profissionais especializados, carecendo de tempo para observação do comportamento, do desenvolvimento das relações sociais e de análise da história de vida. No caso do autismo, mesmo que não exista nenhuma cura conhecida, o diagnóstico precoce e a intervenção imediata favorece a redução de probabilidade de cronificação, a minimização de diversos sintomas e aumenta as possibilidades de tratamento (MAIA, et al., 2016; ABRA, 2013).

O diagnóstico de um transtorno crônico como o autismo, constitui uma situação impactante na vida familiar, visto que, apresenta-se como uma situação que pode propiciar alterações na rotina diária, social, financeiro e na dinâmica familiar, uma vez que necessitam de cuidados continuados para seu desenvolvimento. Zanatta, et al., (2012), enfatiza que a descoberta do diagnóstico crônico incurável, para a família é um processo doloroso de perda, seguindo por um processo de aceitação. Onde a família perde o sonho de ter um filho saudável, independente e se depara com o fato de ter que se reestruturar para promover um auxílio a esse filho para melhoria do seu desenvolvimento.

As dificuldades de cuidar de uma pessoa com autismo se inicia desde a infância, pois sua condição crônica pode afetar a realização de atividades diárias, uma vez que este transtorno pode comprometer as condições físicas e mentais do indivíduo, aumentando a demanda por cuidados, e com isso o nível de dependência de seu cuidador.

Cabe ressaltar que a mãe apresenta-se como principal cuidadora, tendo maior sobrecarga, estando susceptíveis ao desenvolvimento de altos níveis de 
estresse (SCHMIDT; DELL'AGLIO; BOSA, 2007). Estudos têm evidenciado a existência de níveis elevados de estresse familiar e dificuldade emocional em lidar com as situações próprias do autismo relacionadas ao cuidado e acompanhamento a indivíduos com autismo, principalmente nas mães (MISQUIATTI, et al., 2015; SEMENSATO; SCHMIDT; BOSA, 2010; FÁVERO; SANTOS, 2010).

Ainda de acordo com estas pesquisas, aspectos como a sobrecarga de tarefas (a exemplo, dos cuidados com a criança, com a casa e com as consultas), a espera para atendimento, gastos com atendimentos profissionais, pouco ou nenhum espaço para autocuidado, mudanças de rotinas, excesso de responsabilidades, principalmente nas mães, promovem em muitos casos desestabilização, tensão emocional e física nos cuidadores, apresentando-se a necessidade de intervenções que considerem toda a unidade familiar.

Além disso, alguns estudos (GOMES, et al., 2015; FAVERO; SANTOS, 2010; SEMENSATO; SCHMIDT; BOSA, 2010) demonstram correlações entre 0 comportamento das crianças e os níveis de estresse, ansiedade e depressão nos pais. Pesquisa de Misquiatti, et al., (2015) utilizaram grupos comparativos entre familiares de crianças com autista e com transtornos de linguagem a fim de avaliar a sobrecarga de familiares cuidadores, segundo a percepção dos próprios cuidadores, foi constatado que a média do índice de sobrecarga de ambos os grupos foi parecidas, estando moderadamente sobrecarregados.

Estudo realizado por Freitas e Sobrinho (2015), evidenciou que os níveis de estresse e sobrecargas nas mães são altos, e que grupos de apoio social e religioso apresentam-se como estratégias para melhor adaptação destas mães as circunstâncias estressoras. Foi percebido também que as mães conseguem lidar diretamente com o agente estressor advindo do comportamento do filho, no entanto, não sabem lidar com as emoções que surge devido estes comportamentos.

Schmidt, Dell'Aglio e Bosa (2007) identificaram que as mães conseguem lidar diretamente com seus problemas, mas não com as dificuldades dos seus filhos, sugerindo a implementação de grupos de apoio, com foco nas estratégias de enfretamento e que possam auxiliar no alívio do estresse materno e sentimentos advindos do estresse. Diante disto, torna- se importante realizar algumas intervenções juntos aos familiares, como grupos de apoio/suporte familiar, que 
podem minimizar o estresse e promover o autocuidado e a integração entre os familiares.

Segundo Balbino et al., (2015); Alvarez et al., (2012), o grupo de apoio/suporte apresenta-se como um recurso que vem auxiliar no alívio de sentimentos diversos, proporcionando o compartilhamento de experiência, sentimentos e reflexões, e a sensação de ser compreendido. Além disso, ao ofertar apoio emocional, orientação e informação, estes grupos promovem a percepção das vivências, ajudando-os a enfrentar as crises vivenciais. Com base nestas considerações, voltar-se para os familiares é convoca-los, possibilitando com que estes se posicionem diante de si mesmo, do outro, do cuidado e do adoecimento do filho. Este trabalho justifica-se uma vez que compreende e enfatiza os grupos de apoio/suporte como importante para o cuidado, favorecendo o rompimento da lógica de adoecimento voltada apenas para o sintoma e a pessoa doente, o profissional direciona o foco das intervenções para o cuidador e assim promove ações de integralidade.

Em face às elucidações, este relato de abordagem qualitativa descritiva, teve como objetivo descrever a experiência apreendida em um grupo de apoio a familiares de pessoas com autismo, enquanto psicóloga residente em saúde da família e comunidade. Vale salientar que, a experiência vivida nos encontros grupais aconteceu de forma processual, continua e existencial, apesar da descrição e análise desta ter sido sistemática, reflexiva e científica.

Assim, considera-se relevante destacar, sob o aporte teórico da Psicologia da Saúde e das Práticas Integrativas de Cuidado, a importância de grupos de apoio/suporte para os familiares de crianças com autismo, como um espaço de fala com vias a minimização de sobrecarga, troca de experiências e aquisição de conhecimento. 


\section{MÉTODO}

Trata-se de um estudo descritivo, do tipo relato de experiência sobre a atuação enquanto psicóloga residente em saúde da família e comunidade em um grupo de apoio a familiares de pessoas com autismo denominado "Família Azul". Este tipo de estudo possibilita que o profissional em seu cotidiano relate os fenômenos baseados na realidade, relacionando vivências e teorias pertinentes (GIL, 2009; MINAYO, 2013).

O grupo de apoio/suporte é realizado no Centro de Referência Municipal de Inclusão para Pessoas com Deficiência - CRMIPD, localizado no município de João Pessoa - PB. O CRMIPD é um centro que oferece atendimento especializado às pessoas com deficiência, tendo como objetivo a habilitação e a reabilitação de crianças, adolescentes e jovens com deficiência, distúrbio de comportamento e/ou aprendizagem. O grupo "Família Azul" assiste os familiares de pessoas com autismo que são atendidos no CRMIPD.

Cabe ressaltar que neste relato será apresentada de forma sistemática a compilação das experiências vivenciadas a cada encontro com os familiares no grupo. Outrossim, faz-se necessário dizer que todas as práticas de cuidado e de orientação realizada no grupo se objetivaram em procedimentos e intervenções norteadas pelo aporte teórico e metodológico da Psicologia e das Práticas Integrativas em saúde.

Os instrumentos para coleta foram à observação e o diário de campo. Todas as observações, percepções e sentimentos vivenciados pela residente foram anotadas no diário de campo que, num segundo momento serviu de base para refletir sobre cada vivência, a partir das articulações entre teoria e prática. A experiência da escrita no diário de campo configura como uma ferramenta de expressão da experiência, para além dos aparatos técnicos, mas abarcando momentos de angústias e alegrias (DIEHL; MARASCHIN; TITTONI, 2006). Para análise dos dados foi utilizada das anotações do diário de campo, consistindo uma análise qualitativa e contextualizada dos dados obtidos, a partir da análise de conteúdo de Bardin (2011). 


\section{RESULTADOS E DISCUSSÃO}

Atuar em grupos de familiares é compreender autêntica e empaticamente os sentimentos e experiências ali expressos. Concomitantemente, é promover um espaço acolhedor através da construção de vínculos, de práticas de cuidado e de compartilhamentos.

O grupo de apoio/suporte denominado "Família Azul" é realizado no CRMIPD, foi idealizado, em julho de 2017, por um educador físico que atua no CRMIPD, localizado no município de João Pessoa - PB. E para seu desenvolvimento contou com a contribuição e participação de alguns profissionais da referida instituição como educador físico, psicopedagoga e pedagoga e de residentes multiprofissionais em saúde da família e comunidade da Faculdade de Ciências Médica da Paraíba, a saber, psicólogos, enfermeiras, fisioterapeutas, terapeuta ocupacional e farmacêutica.

Os encontros grupais iniciaram-se em agosto de 2017, a qual concilia práticas de cuidado, de orientação, de informação e de apoio. Salienta-se que a atuação enquanto residente multiprofissional em saúde fora realizada desde o início do grupo, agosto de 2017 até novembro de 2017.

Este grupo surgiu pela demanda dos familiares cuidadores de pessoas com TEA por um espaço de apoio, compartilhamento de experiências e saberes. Os familiares procuravam atendimento psicológico com a intenção de cuidar de si, por causa da sobrecarga e estresse advindo do cuidado excessivo e constante que ofertavam as pessoas autistas.

Além disso, os profissionais da referida instituição escutavam angústias, medos e dúvidas dos familiares, impulsionando a criação do grupo. E diante da constante procura de familiares por um espaço de fala, de escuta e de cuidado, estruturou-se o grupo de apoio "Família Azul". Sob este aspecto, Alvarez et al., (2012) afirma que o grupo é uma estratégia que auxilia e fornece suporte frente aos 
diversos sentimentos e vivencias, bem como promove reflexões e a sensação de apoio e compreensão.

Desta forma, o grupo tem como objetivo proporcionar aos familiares cuidadores, através de um processo formativo a possibilidade da construção de conhecimentos, esclarecimento de dúvidas e melhora na autoestima, a partir de rodas de conversa, troca de experiências e cuidados terapêuticos. Bem como, contribuir para uma convivência harmoniosa no cotidiano familiar, compartilhar experiências, a fim de que as próprias famílias se ajudem, articular ações favoráveis às famílias, executar serviços de orientação, apoio, promoção social e viabilizar trabalho de caráter preventivo e transformador.

O grupo é formado por familiares cuidadores de pessoas com autismo, a saber: mães, pais, tias, avós, avôs ou responsável legal de crianças e adolescente com TEA e eram mediados por profissionais do CRMIPD e residentes multiprofissionais de saúde da família e comunidade. Com frequência mensal, duas vezes ao mês, cada encontro grupal era composto por 20 participantes, sua maioria eram as mães, ocorreram 8 encontros grupais totalizando 160 participantes.

Norteado pela premissa de que os familiares buscam um espaço de acolhimento e trocas, eram promovidas ações voltadas para as suas necessidades como: compartilhamento de experiências e saberes, orientações, escutas de apoio, práticas integrativas de cuidado (relaxamento, alongamentos, dança circular, argiloterapia, auriculoterapia) e roda de conversas. Dessa forma, recorreu-se ao uso de práticas de cuidados e de caráter informativa/educativa associada à observação e escuta clínica com a finalidade de compreender os fenômenos psicossociais, emocionais e comportamentais do vivenciar dos familiares cuidadores de pessoas com autismo.

Cada encontro grupal representava um momento de ouvir e, principalmente, ser ouvido em angústias, queixas, desejo, esperanças, anseios, metas. Parecia que estavam ali por causa dos seus familiares com autismo e parecia não se esquecer disto. A todo o momento, observava- se um familiar falando de seu parente com autismo, e os outros escutando. Enquanto psicóloga residente pode-se afirmar o quanto é importante a escuta ativa, onde com o olhar psicológico pôde apreender as singularidades e subjetividades ali expressas. Estudo de Maia et al., (2016) 
evidencia que a escuta estrutura-se como um momento de desabafo do outro e que um grupo voltado para familiares de crianças autistas apresentava-se como uma fonte de apoio e encaminhamentos.

Pode-se afirmar que os familiares ouvem porque se veem naquela narrativa, sentem de forma muito semelhante o sentimento ali expresso, e o respeitam. Toda experiência era única e nada simples, a impressão é que estes familiares ou já viveram aquelas situações expressas ou se preparam para vivencia-las. Diante de todas as falas que ali surgiu, percebe-se que são as trocas de experiências já vividas as que mais ensinam e resignificam as suas atitudes e sentimentos.

Dessa forma, a cada encontro grupal ressonavam vozes de sofrimentos, de superações e de trocas, constituindo-se em cenas de apoio e cuidado, promovendo a percepção de si enquanto sujeito de cuidado. O apoio do grupo apresentou-se como fundamental para ressignificação dessas vozes, e de acordo com Mello et al., (2013), o acolhimento e apoio aos familiares de pessoas com autismo, evita o sofrimento e a solidão, bem como a possibilidade de cooperação e evolução na assistência ao familiar com TEA.

Toda dinâmica familiar se modifica. É comum ouvirmos relatos da necessidade de escuta, de apoio e o grupo vem favorecendo este cuidado. Segundo Pinto et al., (2016); Andrade e Teodoro (2012), o diagnóstico de uma doença crônica impacta a família, promovendo mudanças no cotidiano, nos papéis familiares, no financeiro e em todo o contexto social, além de proporcionar dúvidas e angústias.

Dessa forma, os familiares cuidadores estão no grupo para retirar inquietações e buscar cuidados, percebe-se que os encontros mobilizavam reflexões juntos aos familiares, os convocando a colocar-se no lugar não apenas de quem cuida, mas de quem precisa ser cuidado. Martins (2001) enfatiza que para promover o bem estar das pessoas sob seus cuidados é preciso não se esquecer do nosso próprio.

O grupo representa uma fonte de cuidado, e vem sinalizar aos cuidadores que é necessário também ter espaço e tempo para si, visto que para prestar um cuidado eficaz precisa está bem. Entretanto, durante experiência observou-se que para os familiares, a visão é que quando se tem alguém com alguma deficiência, a família 
vive em função do adoecimento, de suas exigências e limitações, requerendo atenção e dependência permanente.

Hoosteen e Woodgate (2013) evidenciam em pesquisa que os familiares de criança com TEA, centram-se no autismo, colocando suas necessidades em últimos planos, devido à excessiva demanda de cuidados, atenção e dependência. Pode-se afirmar que tais situações apresentam-se como promotora de sobrecargas. Assim, falar em sobrecarga é deparar-se com uma luta constante dos cuidadores de pessoas com autismo, uma vez que todos demonstram enfrentar romarias em busca por profissionais, locais especializados e procedimentos terapêuticos para as pessoas autistas sob seus cuidados.

Além disso, é notório durante experiência que o fato da dependência constante de cuidado, a sobrecarga de tarefas, as mudanças de rotinas e o excesso de responsabilidades apresentam-se para os familiares como fatores que propiciam o adoecimento emocional como o estresse e a ansiedade. Para Fávero e Santos (2005); Andrade e Teodoro (2012), as famílias que cuidam de pessoas especiais, passam por mudanças no cotidiano e na dinâmica familiar, deparando-se com sobrecargas de tarefas e exigências diversas, que podem suscitar em adoecimento emocional.

Em meio aos discursos de sobrecargas e preocupações, recorremos às práticas integrativas e complementares de cuidado no grupo, como meio de ofertar cuidado e promover a minimização destas sensações e sentimentos. Dessa forma, após escuta de suas angústias realizava-se as práticas integrativas. Durante a prática de cuidado como o relaxamento, massagens, auriculoterapia, dança circular, os familiares compreenderam a necessidade de cuidado, do encontro consigo, entrando em harmonia com o corpo e a mente, seguindo o ensinamento do Dr. Edward Bach (SCHEFFER, 2011) do cura-te a ti mesmo.

Neste interim, o grupo "Família Azul" apresenta-se como terapêutico, pois promove um momento para eles mesmos, para refletir sobre si e para cuidados terapêuticos como relaxamentos, reflexões, entre outras. E é nestes momentos de cuidados, que é perceptível o sentimento de acolhimento, onde em meio às emoções que ali surgem, sentem seguros para se expressarem sem ser julgados, entregando ao cuidado. Frente a isto, Schmidt, Dell'Aglio e Bosa (2007) enfatizam que 
o acompanhamento e orientação ao familiar promovem a redução do estresse e a construção de conhecimento.

Mesmo diante das dificuldades e sobrecargas, a impressão que temos é de empoderamento, onde buscam forças e recursos para vencer obstáculos e procurar estratégias para melhorias das limitações das pessoas autistas sob seus cuidados. Pode-se destacar que estes familiares são resilientes. Walsh (2006) corroborando com a ideia de que as famílias podem ter fatores de resiliência, definiu a resiliência familiar como um processo de superação das dificuldades, propiciando o crescimento familiar.

É comum ouvimos relatos, principalmente das mães, de abdicação após o nascimento de seu filho com TEA, tendo em vista que muitas não conseguiam prosseguir com suas carreiras profissionais, devido à demanda de cuidados que as pessoas sob seus cuidados precisam e a falta de outros cuidadores. Corroborando com o exposto Sousa (2011) destaca que devido às demandas de cuidado, as mães vão perdendo sua história e passam a viver a história de seu filho. Alguns autores ainda ratificaram que, na maioria das vezes, são as mães que assumem o papel central de cuidador e de coordenador do tratamento do seu filho (NEALY, et al., 2012).

Foi no grupo que suas faltas, recusas e abdicações eram acolhidas, acalentadas pelos outros familiares. Nestes momentos de fala misturavam se múltiplos sentimentos, que eram elaborados e ressignificados a partir das rodas de compartilhamentos e das práticas integrativas de cuidado, podendo-se afirmar que o grupo tem potencial terapêutico.

Dessa forma, os encontros grupais permitiram identificar os sentimentos mais frequentes dos familiares em relação a cuidar de uma pessoa com autismo, promovendo a abertura de um espaço de fala para minimização e compreensão destes. Os sentimentos de impotência, tristeza, esperança e alegria apareciam com frequência nas falas dos familiares. O sentimento de impotência aparecia associado aos comportamentos da pessoa com autismo, uma vez que expressavam angústias devido às várias tentativas para melhoria dos comportamentos, mas sem êxitos em algumas situações. E diante disso, afirmava haver a sensação de julgamento advindo de outras pessoas ou familiares, relacionado à forma como o cuidador ágil 
frente ao comportamento da pessoa autista sob seus cuidados. Sob este aspecto Semensato, Schimidt e Bosa (2010), evidencia em pesquisa que o sentimento de impotência é frequente em cuidadores de pessoas com TEA, decorrente das dificuldades no manejo dos comportamentos e do entendimento dos mesmos.

Já o de tristeza aparecia associado às sobrecargas no cuidado, principalmente das mães, ao afirmarem não terem um tempo de descanso ou alguém que auxiliassem, visto que demandavam atenção constante. Corroborando com o expresso acima Zanatta et al., (2014), afirma que as mães de crianças com autismo, dedicam-se integralmente a eles, acumulando responsabilidades como o cuidado da família, da casa, e do filho com TEA, o que pode propiciar sobrecargas.

Já o sentimento de alegria associado às superações das dificuldades, diante, por exemplo, da evolução no comportamento das pessoas autistas sob seus cuidados, ao conseguirem assistência à saúde e ao conseguir através deste grupo cuidar-se. E o sentimento de esperança correlaciona-se a assistência que seus familiares com autistas recebem do CRMIPD, uma vez que com os serviços que estes frequentam observam significativa evolução; este sentimento também aparecia embasado na religião, visto que a crença fortalecia-os a seguir a diante.

Outro ponto a se destacar é que o grupo vem favorecendo ainda mais a construção de vínculos entre os familiares e a instituição/profissionais de saúde, além de promover trocas de conhecimento. De acordo com Balbino et al., (2015), grupos de apoio favorecem a aproximação e estreitamento das relações entre os familiares e os profissionais da saúde, promovendo uma relação de confiança e acolhimento, o que faz com que a família sinta-se mais segura e com que o grupo seja uma experiência transformadora.

O grupo também é um espaço em que se possibilita a construção de conhecimento e oferta de informações, permitindo a clarificação e o apaziguamento das dificuldades. $E$ foi no compartilhamento entres os familiares do grupo e os mediadores que se fortaleciam, escutavam exemplos e aprendiam com a história do outro.

E nesta sintonia de construção de conhecimento e acolhimento, que o grupo "Família Azul" se fortalece, apresentando-se como uma das fontes de apoio dos 
familiares cuidadores, frente a essa batalha constante em busca de melhorias para vida de seu familiar com autismo.

Por fim, o grupo constitui um espaço terapêutico de possibilidades, reflexões, trocas e cuidados aos familiares cuidadores, promovendo também vínculos com a instituição e com os outros familiares e gerando o sentimento de pertença e de acolhimento.

\section{CONCLUSÃO}

O diagnóstico de autismo implica mudanças e rupturas avassaladoras para a família, mostrando-se essencial um espaço de fala e escuta, uma vez que é através do ato de compartilhar experiência e construir vivências que os familiares cuidam-se. Assim, o grupo "Família Azul" possibilitou um espaço de cuidado, a partir do compartilhamento de experiência, das práticas de cuidado e da construção de conhecimento mútuo.

Na prática foi possível constatar o quanto o grupo para familiares de pessoas com autismo pode contribuir para minimização das conotações negativas e dos sentimentos advindos das mudanças na dinâmica familiar, financeira e social e da sobrecarga de cuidados. Este grupo favoreceu uma maior compreensão do que se passa com o cuidador, dando voz as contingências e os empoderando em seu cuidado.

Foi possível aprender que o grupo com familiares cuidadores de pessoa com autismo, apresenta-se como um recurso que promove o acolhimento, o suporte das dificuldades diante das limitações das pessoas autistas sob seus cuidados, 0 compartilhamento de múltiplos sentimentos e a interação entre os profissionais da saúde e o cuidador. Dessa forma, verificou- se que o grupo tem potencial terapêutico, pois incita o cuidado e a reflexão.

Assim, a experiência no grupo de apoio foi cheia de significância por proporcionar implicações de forma crítica e reflexiva sobre a prática do psicólogo junto à cuidadores de pessoas com características tão peculiares como o autismo. 
Dessa forma, a atuação neste grupo de apoio vislumbrou possibilidades de atuação do psicólogo, visto que pôde enfatizar a singularidade de cada familiar e a subjetividade do grupo. Além disso, é possível destacar a importância de um trabalho multiprofissional e da realização de práticas integrativas e complementares em saúde em grupos de apoio/suporte.

Grupos de apoio/suporte como recurso terapêutico pode colaborar para uma prática assistencial mais humanizada e acolhedora. E pelos efeitos positivos que 0 grupo promove, é importante que os profissionais da saúde utilizem desta estratégia em suas práticas junto às famílias de pessoas com autismo. Portanto, a experiência proporcionada pelo grupo possibilitou a compreensão da importância de ofertar cuidados para cuidadores, sendo um campo relevante para pesquisas sobre grupos de apoio a familiares cuidadores.

Conclui-se ser o grupo de apoio/suporte uma importante estratégia de cuidado aos familiares de pessoas com autismo, apresentando-se como um recurso a ser utilizado na promoção do cuidado prestado por profissionais de saúde que atuam com cuidadores, com vista à realização de cuidados terapêuticos, educação em saúde e orientações. É um recurso de atenção à saúde a ser utilizada por profissionais que atuam com este público, podendo promover um trabalho mais produtivo e ofertar uma atenção integralizada do cuidado. 


\section{REFERÊNCIAS BIBLIOGRÁFICAS}

ALVAREZ S.Q, et al. Grupo de apoio/ suporte como estratégia de cuidado: importância para familiares de usuários de drogas. Rev Gaúcha Enferm., Porto Alegre (RS) 2012 jun;33(2):102108.

Disponível em:

http://www.seer.ufrgs.br/RevistaGauchadeEnfermagem/article/viewFile/24646/19499>. Acesso em: novembro de 2017.

ANDRADE A. A.; TEODORO, M. L. M. Família e Autismo: Uma Revisão da Literatura. Contextos Clínicos, vol. 5, n. 2, 2012.

ASSOCIAÇÃO BRASILEIRA DO AUTISMO-ABRA. História do autismo (2013). http://autismo.org.br/site/index.php?option=com_content\&view=article\&id=11. Acesso em: fevereiro 2018.

AMERICAN PSYCHIATRY ASSOCIATION. Diagnostic and Statistical Manual of Mental disorders - DSM-5. 5th.ed. Washington: American Psychiatric Association, 2013.

BALBINO, F. S. et al. Grupo de apoio aos pais. Escola Anna Nery Revista de Enfermagem 19(2) Abr-Jun 2015. Disponível em:< http://www.scielo.br/pdf/ean/v19n2/1414-8145-ean-19- 020297.pdf>. Acesso: janeiro de 2018.

BARDIN, Laurence. Análise de conteúdo. São Paulo: Edições 70, 2011, 229 p.

DIEHL, R; MARASCHIN, C; TITTONI, J. Ferramentas para uma Psicologia Social. Psicologia em Estudo, Maringá, v. 11, n. 2, 2006, p. 407-415. Disponível em: <http://www.scielo.br/pdf/pe/v11n2/v11n2a19>. Acesso em: novembro de 2017.

FÁVERO, M. A.; SANTOS, M. A. Autismo Infantil e Estresse Familiar: uma revisão sistemática da literatura. Psicologia Reflexão e Crítica, 2005, 18(3), pp. 358-369.

FAVERO-NUNES, M. A.; SANTOS, M. A. dos. Itinerário terapêutico percorrido por mães de crianças com transtorno autístico. Psicol. Reflex. Crit. [online]. 2010, vol.23, n.2, pp.208-221. ISSN 0102-7972. Disponível em: <http://dx.doi.org/10.1590/S0102-79722010000200003>. Acesso em: dezembro de 2017.

FREITAS, S.S; SOBRINHO, L.A. Estratégias de enfrentamento de mães com filhos autistas. Trabalho de Conclusão de curso. Faculdade Castro Alves, Salvador, 2015.

FEDERAÇÃO NACIONAL DAS APAES. Número de pessoas com autismo aumenta em todo - Brasil. 2017. Disponível em: <https://apaebrasil.org.br/noticia/numero-de-pessoas- comautismo-aumenta-em-todo-o-brasil>. Acesso em: novembro de 2017.

GIL A. C. Métodos e técnicas de pesquisa social. São Paulo: Atlas, 2009.

GOMES, P.T.M. et al,. Autismo no Brasil, desafios familiares e estratégias de superação: revisão sistemática. Jornal de Pediatr. (Rio J.) vol.91 n.2 Porto Alegre Mar./Abr. 2015. Disponível em: < http://www.scielo.br/scielo.php?pid=S002175572015000200111\&script=sci_arttext\&tlng=pt>. Acesso em: novembro de 2017.

HOOGSTEEN, L. WOODGATE, R. Centering Autism Within the Family: A Qualitative Approach to Autism and the Family. Journal of Pediatric Nursing, 28, 2013, 135-140.

MAIA, F. A. et al,. Importância do acolhimento de pais que tiveram diagnóstico do transtorno do espectro do autismo de um filho. Cad. Saúde Colet., 2016, Rio de Janeiro, 24 (2): 228-234. 
MARTINS, M. C. F. Humanização das relações assistenciais de saúde: a formação do profissional de saúde. São Paulo: Casa do Psicólogo, 2001.

MELLO, A.M.S.R. de. Autismo: guia prático. 5 ed. São Paulo: AMA; Brasília: CORDE, 2007. 104 p.: il.

MELLO, A. M. S. R. de, et al. Retratos do autismo no Brasil, $1^{\text {a }}$ ed. São Paulo: AMA, 2013.

MINAYO, M. C. S. O desafio do conhecimento: pesquisa qualitativa em saúde. 13. ed., São Paulo: Hucitec, 2013.

MISQUIATTI, ARN. Sobrecarga familiar e crianças com transtornos do espectro do autismo: perspectiva dos cuidadores. Rev. CEFAC. 2015 Jan-Fev; 17(1):192-200. Disponível em:< http://www.scielo.br/pdf/rcefac/v17n1/1982-0216-rcefac-17-01-00192.pdf>. Acesso em: novembro de 2017.

NEALY, C., et al. (2012). The Impact of Autism Spectrum Disorders on the Family: A Qualitative Study of Mothers' Perspectives. Journal of Family Social Work, 15, 187-201.

PINTO, R.N.M. et al. Autismo infantil: impacto do diagnóstico e repercussões nas relações familiares. Rev Gaúcha Enferm. 2016 set;37(3):e61572. Disponível em:< http://www.scielo.br/pdf/rgenf/v37n3/0102-6933-rgenf-1983-144720160361572.pdf>. Acessado em: novembro de 2017.

SCHEFFER, M. Terapia floral do Dr. Bach: teoria e prática. 13. ed. São Paulo: Pensamento, 2011.

SCHMIDT, C.; DELL'AGLIO, D. D.; BOSA, C. A. Estratégias de Coping de Mães de Portadores de Autismo: Lidando com Dificuldades e com a Emoção. Psicologia Reflexão e crítica, Porto Alegre, V.20, n.1, p.124-131, 2007.

SEMENSATO, M.; SCHMIDT, C.; BOSA, C. A. Grupo de familiares de pessoas com autismo: relatos de experiências parentais. Aletheia, núm. 32, 2010, pp. 183-194.

SOUSA, L. A. A Interação da Família com a Criança Autista. Trabalho de Conclusão de Curso (Graduação em Psicologia) - Faculdade Integral Diferencial. Teresina-Piauí, 2011.

WALSH, F. Strengthening Family Resilience. The Guilford Press. 2006, 2nd Ed.

ZANATTA, E. A., et al. Cotidiano de famílias que convivem com o autismo infantil. Revista Baiana de Enfermagem, Salvador, v. 28, n. 3, p. 271-282, 2012. 